\title{
Investigation on the Status Quo of Self-Health Management of Patients with Bipolar Disorder and Analysis of Influencing Factors
}

\author{
Shan Cai, Lin Zhou, Xiao Yang, Chenyu Ma, Liuliu Xu $(\mathbb{D}$, and Ruilian Qian \\ The Affiliated Brain Hospital of Nanjing Medical University, China \\ Correspondence should be addressed to Liuliu Xu; caishan@njmu.edu.cn and Ruilian Qian; 2209188046@qq.com
}

Received 10 March 2021; Revised 18 March 2021; Accepted 24 March 2021; Published 24 May 2021

Academic Editor: Songwen Tan

Copyright @ 2021 Shan Cai et al. This is an open access article distributed under the Creative Commons Attribution License, which permits unrestricted use, distribution, and reproduction in any medium, provided the original work is properly cited.

\begin{abstract}
In this study, we aim to explore the status quo of self-health management of patients with bipolar disorder and influencing factors. A total of 80 patients with bipolar disorder were included and assigned into the observation group and the control group, with 40 cases per group. The patients in the control group were given general health education, and those in the observation group were given personalized education combined with peer support. It was found that there was no significant difference in gender, age, educational degree, marital status, and living state between the observation group and the control group $(P>0.05)$. There are significant differences with regard to self-management between the two groups. The observation group showed stronger self-care ability, health education level, self-protection ability, and emotional control ability than the control group $(P<0.05)$. Before the intervention, no significant difference in Hamilton Depression Rating Scale (HAMD) scores was observed between the observation group and the control group $(P>0.05)$. After the intervention, the HAMD scores were lower in the observation group than in the control group $(P<0.05)$, suggesting an alleviation in manic and depressive episodes in the observation group. It was also revealed that the observation group exhibited lower Functioning Assessment Short Test (FAST) scores in all aspects than the control group, suggesting personalized education combined with peer support could significantly improve the quality of life of patients with bipolar disorder. Self-health management ability of the patients was regarded as the dependent variable and healthy education level, health literacy, and objective support as the independent variables, and the regression model was performed. The results show that information acquisition ability, communication and interaction ability, objective social support, subjective social support, and utilization of social support can all affect the self-health management ability of patients. Personalized education combined with peer support can effectively alleviate the condition of patients with bipolar disorder, improve the degree of overall functional impairment, enhance the patient's self-management ability, and promote their recovery. It is worthy of promotion and application. In conclusion, information acquisition ability, communication and interaction ability, objective social support, subjective social support, and utilization of social support can all affect the self-health management ability of patients.
\end{abstract}

\section{Background}

Bipolar disorder refers to a mood disorder that has both manic and depressive episodes [1]. The clinical manifestations are repeated alternating manic episodes and depressive episodes or a mixed episode of both [2]. Bipolar disorder is a complicated mental disorder caused by a multifactor genesis with both biological-genetic and psychosocial factors [3]. The disorder was characterized by limited treatment, high recurrence rate, and suicide rate $[4,5]$. Because bipolar disorder can damage the cognitive function and social function of patients, it will not only affect the patient's own quality of life but also bring a heavy burden to their family and society $[6,7]$. The Hamilton Depression Rating Scale (HAMD) is a clinician-rated instrument that is considered standard scales for evaluating depressive symptoms worldwide [8]. The Functioning Assessment Short Test (FAST) is a brief instrument designed to assess the main functioning problems experienced by psychiatric patients, particularly bipolar patients, which consists of 24 items that assess impairment or disability in six specific areas of functioning: autonomy, occupational functioning, cognitive functioning, financial issues, interpersonal relationships, and leisure time [9]. 
Studies have found that personalized education combined with peer support can improve the patient's condition and enhance their overall functions and self-management capabilities $[10,11]$, but this conclusion remains to be verified. Self-health management refers to the whole process of analyzing, predicting, and preventing one's own health information and risk factors. The management method is to monitor your own health information at any time with the help of health scales, health assessment software, or health information systems [12]. It is an entry point to maximize the effect of health management for patients with bipolar disorder, which can help patients establish correct health management beliefs, improve health literacy, and prompt the elderly to take responsibility for their health. This study intends to use health education or personalized education combined with peer support to observe differences in patients' selfhealth management capabilities and their influencing factors.

\section{Materials and Methods}

2.1. Clinical Data. Eighty patients with bipolar disorder who were admitted to the hospital from January 2019 to January 2020 were selected and divided into the observation group and the control group according to different nursing methods. The control group and the observation group each had 40 cases. This study was completed under the supervision and approval of the peer support service supervision group. Inclusion criteria are as follows: (1) initial diagnosis of bipolar disorder according to the $10^{\text {th }}$ edition of the International Classification of Diseases for bipolar disorder (PMID: 32447353), (2) with a junior high school education or above and ability to receive personalized education and peer support, (3) ability to answer the questionnaire survey, and (4) an informed consent available from patients or family members. Exclusion criteria are as follows: (1) those with other serious mental illnesses, (2) those with severe physical illnesses, (3) those with mental disorders caused by alcohol and drugs, and (4) those with hearing impairments or language disorders that are difficult to communicate effectively.

2.2. Method. The control group used general health education, and the observation group used personalized education combined with peer support.

2.2.1. Personalized Education. Formulation of education plan: evaluate the patient's personal situation and formulate a personalized education plan based on the patient's condition, personality, education level, family situation, and living environment.

Health education: it is possible to explain the causes of bipolar disorder, treatment plan, usage and dosage of drugs, and adverse reactions to patients and their families through oral education, health manuals, and health knowledge lectures and what needs attention in life. It emphasizes the importance of taking medication as prescribed and guides them to develop a regular and healthy lifestyle. At the same time, they teach family members how to deal with the patient's illness so that they can effectively deal with the patient's condition.
Psychological education: the psychology of bipolar disorder is relatively fragile. Therefore, when communicating with patients, you should show enthusiasm and respect and listen patiently to what the patients say so that they can express their emotions, find out the crux of the problem, and provide them with psychological guidance, such as guiding them to express anger and vent their emotions and improve their ability to cope with conflicts, so as to be able to handle interpersonal relationships and improve self-protection.

2.2.2. Peer Support. Recruitment of counselors. We first extended the peer support service to various clinical departments, which aroused a great response. Many patients actively signed up and asked to participate in the peer support service. Finally, according to the abovementioned conditions required for service development, after evaluation, we selected qualified peer counselors from some active participants.

Implementation method. The implementation of peer support is divided into separate activities in closed wards and open wards, and the activities of each phase are attended by two counselors and two supervisors. We have developed a flow of activities. Before the start of the activity, we will make full preparations. We will work out the theme with the counselor in the previous week, prepare the materials needed for the activity, meet with the counselor and supervisor of related activities, discuss the process of the activity and possible emergencies, and get ready. Go through the process 40 minutes before the start of the event to ensure that the event goes smoothly. The process of the activity is that the two counselors first introduce themselves and then the counselors explain the theme, significance, and precautions of the activity, emphasize the principle of confidentiality, read the counselor's declaration together, and the recovered people introduce themselves and know each other. Warm-up activity: the counselor shared the meaning of the warm-up activity at a turning point and transferred to the theme activity. After the activity, everyone's sharing session. Finally, the counselor summarized and sang our garden song and thanked everyone for their participation and support to the service activities with the supervisor. Another of our counselors will record the entire activity and finally leave the image data.

2.3. Observation Indicators. HAMD score. The YMRS scale and the HAMD scale were used to evaluate the patients' manic and depressive episodes. The higher the score, the more serious the condition. The HAMD scores before and after the intervention were compared between the two groups.

FAST score. The FAST scale was used to evaluate the patients' overall functional impairment from the aspects of autonomous life, occupational function, interpersonal relationship, leisure, etc., and the FAST scores of the two groups after intervention were compared.

Self-management level. The self-care ability, health education level, self-protection ability, emotional control ability, and other self-management levels after intervention were compared between the two groups. 
TABLE 1: Comparison of general information of the two groups of patients.

\begin{tabular}{|c|c|c|c|c|}
\hline & Control group $(n=40)$ & Observation group $(n=40)$ & $X^{2}$ & $P$ value \\
\hline Gender (male/female) & $18 / 22$ & $19 / 21$ & 0.68 & 0.25 \\
\hline Age & $35.68 \pm 5.76$ & $35.76 \pm 5.89$ & 1.36 & 0.69 \\
\hline \multicolumn{5}{|l|}{ Marital status } \\
\hline Unmarried & 12 & 13 & \multirow{4}{*}{2.65} & \multirow{4}{*}{0.16} \\
\hline Married & 25 & 23 & & \\
\hline Divorced & 2 & 2 & & \\
\hline Death of a spouse & 1 & 2 & & \\
\hline \multicolumn{5}{|l|}{ Level of education } \\
\hline Junior high school and below & 7 & 6 & \multirow{3}{*}{1.67} & \multirow{3}{*}{0.23} \\
\hline High school and technical secondary school & 18 & 17 & & \\
\hline College degree or above & 15 & 17 & & \\
\hline \multicolumn{5}{|l|}{ Living state } \\
\hline Living alone & 16 & 18 & \multirow[t]{2}{*}{1.65} & \multirow[t]{2}{*}{0.38} \\
\hline Not living alone & 24 & 22 & & \\
\hline
\end{tabular}

2.4. Statistical Analysis. The SPSS 22.0 software was used to process the count data, the $\mathrm{c} 2$ test was performed using $n(\%)$, and the measurement data was performed using the $t$-test, which was represented by mean \pm standard deviation. The difference was statistically significant at $P<0.05$.

\section{Results}

3.1. Comparison of General Information of Patients with Bipolar Disorder. There were 80 patients with bipolar disorder included in the study, and they were split into the observation and control groups. It was found that there was no significant difference in gender, age, educational degree, marital status, and living state between the two groups of patients $(P>0.05)$; see Table 1 for details.

3.2. Comparison of Self-Management Levels before and after Intervention between the Two Groups. Through comparison, it is found that there are significant differences in selfmanagement levels between the two groups of patients after intervention. After intervention, the self-management level of patients in the observation group, such as self-care ability, health education level, self-protection ability, and emotional control ability, was stronger than that of the control group. The difference was statistically significant $(P<0.05)$; see Table 2 for details.

3.3. Comparison of HAMD Scores before and after Intervention between the Two Groups. After comparing the HAMD scores before and after the intervention of the two groups, it was found that there was no statistical difference in the scores before the intervention $(P>0.05)$, while the HAMD scores of the observation group after the intervention were lower than those of the control group, and the improvement of manic and depressive episodes in the observation group was strong. In the control group, the difference was statistically significant $(P<0.05)$; see Table 3 for details.

3.4. Comparison of FAST Scores between the Two Groups of Patients after Intervention. By observing the patient's auton- omous life, occupational function, interpersonal relationship, leisure, and other aspects, the overall functional impairment of the patient was evaluated, and the FAST score was performed. The results showed that the scores of the observation group were lower than those of the control group in all aspects, suggesting that the observation group has a good treatment effect; see Table 4 for details.

3.5. Multifactor Analysis of Patients' Self-Health Management Ability. We use the patient's self-health management ability as the dependent variable and the statistically significant data in the single factor analysis as the independent variable and perform multiple stepwise regression analysis. Health education level, health literacy, objective support, etc. enter the regression model. The results show that information acquisition ability, communication and interaction ability, objective social support, subjective social support, and utilization of social support can all affect the self-health management ability of patients; see Table 5 for details.

\section{Discussion}

Bipolar disorder is a common clinically severe mental disorder. In the intermittent period, patients completely deal with the normal state, but in the onset period, patients may experience emotional instability, inattention, irritability, substance abuse, and suicidal ideation. Due to the high recurrence rate of the disease, drug treatment and psychotherapy are indefinitely needed to reduce the risk of recurrence [13]. However, some patients lack sufficient knowledge of the disease, which results in pessimistic and desperate psychology. In addition, some patients lack understanding of the degree of harm of the disease, which leads to a decline in selfmanagement ability after discharge from the hospital and a decrease in medication compliance. Standard health education is only conducted through oral education by nursing staff [14]. Although it can meet the basic needs of patients during treatment in the hospital, it cannot take into account the personal situation of each patient. As a result, patients 
TABLE 2: Comparison of self-management levels between the two groups of patients after intervention.

\begin{tabular}{lcccc}
\hline Group & Self-care ability & Health education level & Self-protection ability & Emotional control \\
\hline Control group $(n=40)$ & $61.23 \pm 5.69$ & $69.65 \pm 3.39$ & $62.38 \pm 7.59$ & $58.67 \pm 3.85$ \\
Observation group $(n=40)$ & $84.68 \pm 6.85$ & $89.62 \pm 5.87$ & $87.65 \pm 6.58$ & $76.58 \pm 4.52$ \\
$T$ value & 16.35 & 14.68 & 13.65 & 16.59 \\
$P$ value & $\leq 0.001$ & $\leq 0.001$ & $\leq 0.001$ & $\leq 0.001$ \\
\hline
\end{tabular}

TABLE 3: Comparison of HAMD scores between the two groups before and after intervention.

\begin{tabular}{lcc}
\hline Group & $\begin{array}{c}\text { Before the } \\
\text { intervention }\end{array}$ & $\begin{array}{c}\text { After the } \\
\text { intervention }\end{array}$ \\
\hline Control group & $23.65 \pm 3.59$ & $15.67 \pm 2.15$ \\
Observation group & $23.69 \pm 2.89$ & $9.78 \pm 1.59$ \\
$T$ value & 0.15 & 10.36 \\
$P$ value & 0.64 & $\leq 0.001$ \\
\hline
\end{tabular}

and their families do not fully understand the content of health education, which is not conducive to disease. Therefore, it is necessary to strengthen the health education of patients in order to enhance the patients' awareness of the disease and improve the patients' self-management ability and disease control effect.

This study implemented personalized education and peer support for patients with bipolar disorder and achieved ideal results. In this study, the HAMD scores of the observation group and the control group after intervention were lower than those before the intervention, and the improvement of manic episodes and depressive episodes in the observation group was better than that of the control group. Personalized education combined with peer support for patients with bipolar disorder can alleviate the symptoms of patients and help improve the prognosis of patients. Individualized education can formulate targeted education programs to fit each patient's cognition and understanding ability so that patients and their families can better master the content of health education. It not only can improve the patients' self-care awareness and the family's ability to respond to the patient's condition but also enable patients to master the methods of emotional control and catharsis at the onset of disease, improve the patients' unhealthy psychology through psychological education, enhance the enthusiasm of patients, and enable them to actively participate in treatment and nursing $[15,16]$. However, the use of personalized education alone has limited impact on health education and selfmanagement after discharge from the hospital [17]. Peer support means that people who have faced, encountered, and overcome misfortunes and disasters can provide useful support, encouragement, and hope and even become mentors and friends for those facing the same situation [18]. Peers are organized with each other through interaction between group members to guide and help each other. Group members have no hierarchy among each other, can listen to each other, discuss problems, share patient knowledge and experience that many medical staff do not have, and increase their sense of social role. Since group members are all patients with bipolar disorder, they are more likely to resonate and communicate more naturally [19].

In addition, peer support has the following advantages for patients with bipolar disorder: for patients, it can improve curative effect and reduce relapse and can improve the social support, social function, quality of life, and service satisfaction of patients with severe mental illness; for peers, the social role of "service providers" greatly enhances their sense of self-efficacy, making them feel fulfillment and their own value, thereby enhancing self-confidence and self-esteem. Patients with mental illness often pay more attention to themselves. Less caring about others, peer work has helped them achieve a leap in their roles. Peer service allows recovered patients to experience the connotation of work and is also a transition for them to engage in other jobs in the future. It is beneficial to its continuous recovery; for medical workers, companions can act as a bridge between medical workers and patients, explain the doctor's advice to the patient, convey the patient's intentions and ideas to the doctor, and enhance the doctor-patient relationship. Mutual understanding between the two can improve patient compliance and improve curative effect. As far as the general public is concerned, there has been discrimination against patients with mental illness for a long time, and the public's awareness of mental illness is very low. The survey in some areas in 2005 showed that it was only $30 \%-40 \%$. Peer support allows recovered patients to provide services to others, participate in the work of popularizing mental illness knowledge, and affirm that they can support themselves from the perspective of social roles and are people who contribute and are valuable to society. This enables the public to wait for them equally, understand that mental illness can be cured clinically, and strengthen the public's awareness of severe mental illness, thereby eliminating the fear of patients with mental illness, diminishing discrimination, and reducing the stigma of patients with mental illness. Conversely, social acceptance of patients with mental illness can also have a positive impact on their recovery, which is more conducive to the return of patients with mental illness to society. In this study, the scores of all FAST dimensions such as autonomous life, occupational function, interpersonal relationship, and leisure in the observation group were lower than those in the control group. In addition, choose patients with stable illness, high education, strong communication skills, and cheerful personality as the team leader so that they can lead by example, set an example for the team members, and supervise and help the team members, which will help improve the team members' self-management ability. In this study, the 
TABLE 4: Comparison of FAST scores between the two groups of patients after intervention.

\begin{tabular}{lcccc}
\hline Group & Independent life & Professional ability & Interpersonal relationships & Leisure \\
\hline Control group & $1.89 \pm 0.35$ & $7.85 \pm 0.95$ & $8.65 \pm 1.29$ & $1.98 \pm 0.43$ \\
Observation group & $1.35 \pm 0.58$ & $5.86 \pm 0.86$ & $6.28 \pm 1.16$ & $1.46 \pm 0.37$ \\
$T$ value & 5.89 & 10.36 & 14.68 & 8.79 \\
$P$ value & 0.010 & $\leq 0.001$ & 0.020 & $\leq 0.001$ \\
\hline
\end{tabular}

TABLE 5: The correlation between patients' self-health management ability, health literacy, and social support.

\begin{tabular}{lcc}
\hline Project & $R$ & $P$ \\
\hline Information acquisition ability & 0.36 & 0.001 \\
Communication and interaction ability & 0.29 & 0.001 \\
Objective social support & 0.28 & $\leq 0.001$ \\
Subjective social support & 0.34 & 0.010 \\
Utilization of social support & 0.18 & 0.010 \\
\hline
\end{tabular}

observation group's self-care ability, health education level, self-protection ability, emotional control ability, and other self-management levels after intervention were better than those of the control group. It can be seen from this that the use of personalized education combined with peer support in patients with bipolar disorder can help improve the patient's self-management ability and facilitate the early recovery of patients. In this study, personalized education combined with peer support can effectively alleviate the condition of patients with bipolar disorder, improve the degree of overall functional impairment, enhance the patient's selfmanagement ability, and promote their recovery. It is worthy of popularization and application.

In this study, by comparing the general information of the two groups of patients, it was found that there was no significant difference in gender, age, and educational level between the two groups of patients. Through comparison, it is found that there are significant differences in selfmanagement levels between the two groups of patients after intervention. After the intervention, the self-management level of patients in the observation group, such as self-care ability, health education level, self-protection ability, and emotional control ability, was stronger than that of the control group, and the difference was statistically significant. After comparing the HAMD scores before and after the intervention of the two groups, it was found that there was no statistical difference in the scores before the intervention, while the HAMD scores of the observation group after the intervention were lower than those of the control group, and the improvement of manic and depressive episodes in the observation group was strong than that in the control group. The FAST score was carried out to evaluate the overall functional impairment of the patient by observing the patient's autonomous life, occupational function, interpersonal relationship, leisure, and other aspects. The results showed that the scores of the observation group were lower than those of the control group in all aspects, suggesting that the observation group has a good treatment effect. We use the patient's self-health management ability as the dependent variable and the statistically significant data in the single factor analysis as the independent variable and perform multiple stepwise regression analysis. Education level, health literacy, objective support, etc. enter the regression model. The results showed that information acquisition ability, communication and interaction ability, objective social support, subjective social support, and utilization of social support can all affect the self-health management ability of patients.

In summary, personalized education combined with peer support can effectively alleviate the condition of patients with mental disorders, improve the degree of overall functional impairment, enhance the patient's self-management ability, and promote their recovery. It is worthy of promotion and application. In addition, information acquisition ability, communication and interaction ability, objective social support, subjective social support, and utilization of social support can all affect the self-health management ability of patients.

\section{Data Availability}

The data used to support the findings of this study are included within the article.

\section{Conflicts of Interest}

The authors declare that they have no conflicts of interest.

\section{Acknowledgments}

The study was supported by the Study on Education in the Fourth Clinical Medical College of Nanjing Medical University in 2019 (SLYB2019-01).

\section{References}

[1] L. Tondo, G. H. Vazquez, and R. J. Baldessarini, "Depression and mania in bipolar disorder," Current Neuropharmacology, vol. 15, no. 3, pp. 353-358, 2017.

[2] J. K. Muller and F. M. Leweke, "Bipolar disorder: clinical overview," Medizinische Monatsschrift für Pharmazeuten, vol. 39, no. 9, pp. 363-369, 2016.

[3] N. Freund and G. Juckel, "Bipolar disorder: its etiology and how to model in rodents," Methods in Molecular Biology, vol. 2011, pp. 61-77, 2019.

[4] E. M. Camacho, D. Ntais, S. Jones et al., "Cost-effectiveness of structured group psychoeducation versus unstructured group support for bipolar disorder: results from a multi-centre 
pragmatic randomised controlled trial," Journal of Affective Disorders, vol. 211, pp. 27-36, 2017.

[5] J. N. Miller and D. W. Black, "Bipolar disorder and suicide: a review," Current Psychiatry Reports, vol. 22, no. 2, p. 6, 2020.

[6] B. Sole, E. Jimenez, C. Torrent et al., "Cognitive impairment in bipolar disorder: treatment and prevention strategies," The International Journal of Neuropsychopharmacology, vol. 20, no. 8, pp. 670-680, 2017.

[7] C. D. M. Bonnin, M. Reinares, A. Martinez-Aran et al., "Improving functioning, quality of life, and well-being in patients with bipolar disorder," The International Journal of Neuropsychopharmacology, vol. 22, no. 8, pp. 467-477, 2019.

[8] A. M. Carneiro, F. Fernandes, and R. A. Moreno, "Hamilton depression rating scale and Montgomery-Asberg depression rating scale in depressed and bipolar I patients: psychometric properties in a Brazilian sample," Health and Quality of Life Outcomes, vol. 13, no. 1, p. 42, 2015.

[9] A. R. Rosa, J. Sánchez-Moreno, A. Martínez-Aran et al., "Validity and reliability of the functioning assessment short test (FAST) in bipolar disorder," Clinical Practice and Epidemiology in Mental Health, vol. 3, no. 1, p. 5, 2007.

[10] F. Lobban, N. Akers, D. Appelbe et al., "A web-based, peersupported self-management intervention to reduce distress in relatives of people with psychosis or bipolar disorder: the REACT RCT," Health Technology Assessment, vol. 24, no. 32, pp. 1-142, 2020.

[11] J. A. Cook, M. E. Copeland, J. A. Jonikas et al., "Results of a randomized controlled trial of mental illness selfmanagement using wellness recovery action planning," Schizophrenia Bulletin, vol. 38, no. 4, pp. 881-891, 2012.

[12] C. A. Janney, M. S. Bauer, and A. M. Kilbourne, "Self-management and bipolar disorder-a clinician's guide to the literature 2011-2014," Current Psychiatry Reports, vol. 16, no. 9, p. 485, 2014.

[13] J. R. Geddes and D. J. Miklowitz, "Treatment of bipolar disorder," Lancet, vol. 381, no. 9878, pp. 1672-1682, 2013.

[14] D. A. Perlick, C. Jackson, S. Grier et al., "Randomized trial comparing caregiver-only family-focused treatment to standard health education on the 6-month outcome of bipolar disorder," Bipolar Disorders, vol. 20, no. 7, pp. 622-633, 2018.

[15] M. P. DelBello, "Treating bipolar disorder in pediatric patients and educating patients and parents," The Journal of Clinical Psychiatry, vol. 80, no. 1, 2019.

[16] F. Rouillon, I. Gasquet, R. P. Garay, and S. Lancrenon, "Impact of an educational program on the management of bipolar disorder in primary care," Bipolar Disorders, vol. 13, no. 3, pp. 318-322, 2011.

[17] F. Gumus, S. Buzlu, and S. Cakir, "Effectiveness of individual psychoeducation on recurrence in bipolar disorder; a controlled study," Archives of Psychiatric Nursing, vol. 29, no. 3, pp. 174-179, 2015.

[18] J. F. Kelly, L. Hoffman, C. Vilsaint, R. Weiss, A. Nierenberg, and B. Hoeppner, "Peer support for mood disorder: characteristics and benefits from attending the Depression and Bipolar Support Alliance mutual-help organization," Journal of Affective Disorders, vol. 255, pp. 127-135, 2019.

[19] B. Puschner, J. Repper, C. Mahlke et al., "Using peer support in developing empowering mental health services (UPSIDES): background, rationale and methodology," Annals of Global Health, vol. 85, no. 1, 2019. 